\title{
Adoption Issues for Cloud Computing
}

\author{
Won $\mathrm{Kim}^{1}$, Soo Dong Kim ${ }^{2}$, Eunseok Lee ${ }^{3}$, Sungyoung Lee \\ ${ }^{1}$ Kyungwon University, ${ }^{2}$ Sungsil University \\ ${ }^{3}$ Sungkyunkwan University, ${ }^{4}$ Kyunghee University
}

S. Korea

\begin{abstract}
Cloud computing allows users to use only a Web browser to receive computing services via the Internet. Users only need to pay for the services they actually use. It appears that a wide adoption of cloud computing in the foreseeable future is inevitable, and its adoption will bring about a sea change in the pricing and distribution practices for both software and hardware. There are, however, various issues that will impede adoption of cloud computing. Most of them can be solved. We discuss the status of cloud computing today and various adoption issues. We also provide a market prognosis.
\end{abstract}

\section{Introduction}

Cloud computing is already being used by tens of millions of people in various manifestations, including free email services, such as Yahoo Mail, Hot Mail, Gmail, etc.; and free office productivity applications, such as Google Apps; and numerous subscription-based software as a service (SaaS) services. The term 'cloud' in cloud computing means a 'remote data center'. Cloud computing has a two-part definition [Naone 07][Hoover 08a][Kim 09][wikipedia]. The first is access, via the Internet using a Web browser, to computing resources that are administered remotely and are dynamically allocated and deallocated according to the needs of the users. The second is paying for the actual use of the computing resources. The ability to dynamically allocate and deallocate computing resources means the users do not need to procure servers and storage for peak time use, and let those servers and storage go unused most of the time. The ability to access computing resources outside the users' premises means the users do not need to maintain the computing resources, allocate space to house the computing resources, pay for electricity, and pay for the staff to administer the computing resources. 
We expect that cloud computing, if it takes root, will have profound impact on the IT landscape as we know it today. First, as many types of popular software are served out of the cloud, and software upgrade is done in the cloud, a drastic change to the way software is distributed and priced is inevitable. Software vendors charge license fees for the use of their software, and, in the case of enterprise software, charge annual maintenance fees. They also charge for training and consulting on the use of software. This way of doing business will not be viable for many types of software. Second, as the client computers will no longer need to keep some of the software, they are likely to become lighter and less expensive. The move towards cloud computing is likely to accelerate the adoption of netbooks. Third, cloud computing will create a new IT ecosystem. Some of the laaS service providers will host some of the SaaS, CaaS and PaaS service providers. Cloud management service providers will support other cloud service providers.

In Section 2, we will discuss the reasons for the emergence of cloud computing, and discuss. In Section 3, we will examine adoption issues for cloud computing. Then in Section 4, we will conclude the paper by providing a market prognosis for cloud computing.

\section{Reincarnation}

As a concept, cloud computing is not entirely new. It is similar to the time-sharing systems of the 1960s, and the network computing and grid computing of the 1990s. Further, such services as free email services, Internet search services, Internet portal services, many SaaS services, web hosting services, and some computing infrastructure services, etc. are all cloud computing services that predate the coining of the term cloud computing. In our view, there are four key reasons there is a renewed push and interest in cloud computing these past few years. They include advances in virtualization technologies, Web technologies, scale-out technologies, and infrastructure hardware and software technologies.

Virtualization technologies, such as VMware and open source Xen, now make it possible for data centers to run more than one operating system on a single server; pool together many servers into a single virtual server. Many hard disk drives can be pooled together into a single storage area network. Main memories of commodity x 86 computers can be pooled together into a large-capacity memcache; and networking bandwidth can be pooled together and then split it into independent channels.

Web technologies include Web servers, Web programming languages (HTML, CSS, PHP, JSP, ...), communication protocols (HTTP, SHTTP, ...), and various standards. 
The scale-out technologies include file systems, such as Google File System and Hadoop; distributed and replicated databases; and parallelization technology, such as MapReduce - all for a massive network of servers and storage. The advances in the scale-out technologies have been led by three types of companies: Internet companies, such as Google, Yahoo, Microsoft, MySpace, Facebook, etc.; enterprise software and services companies, such as IBM, Oracle, HP, Microsoft, etc.; and computing infrastructure services companies, such as RackSpace, Iron Mountain Digital, etc.

Advances in infrastructure technologies include both hardware and software. Hardware include servers, storage, and networking. Software include application development environment; and application runtime support, including application frameworks and application servers. The availability of relatively mature and reliable open source software of all types is also an important consideration in the emergence and adoption of cloud computing.

Several types of cloud computing services are available today, including SaaS, infrastructure as a service (laaS), platform as a service (PaaS), component as a service (CaaS), and cloud management services [Gruman 08][Kim 09]. Some SaaS services are for consumers, while others are for enterprises. laaS services provide provisioned virtual machines and storage. PaaS services provide an application development environment and hosts the applications. CaaS services provide an API to Web services to make use of the Web services.

Today, cloud computing has widest uses in Web site hosting, software testing, and online data backup. New software testing often requires additional servers and storage. The servers and storage may be expensive. It usually takes a cumbersome internal process and a fairly long time to procure the computing resources. Further, after testing, the computing resources may no longer be necessary and have to be returned or dispensed with. It is easier, faster, and relatively less expensive to buy a limited-time use of the additional computing resources from an laas service provider.

\section{Adoption Issues}

There are three types of potential users of cloud computing services: consumers, small organizations, and medium to large organizations. Consumers and small organizations have relatively simpler requirements for adopting a new technology than medium to large organizations, and have much less to lose if the adoption goes awry. There are at least seven types of adoption issues for cloud computing [blogspot][Kim 09]. These include outage (availability), security, performance, compliance, private clouds, integration, and cost. Only 
some of them matter to consumers and small organizations. However, all of them are of concern to medium to large organizations.

\section{Outage}

An outage may be temporary and permanent. A permanent outage occurs when a cloud service provider goes out of business. This has happened, and will happen again. Temporary outage of cloud computing services appears to be inevitable. It may happen several times a year, and each time it may last a few hours or nearly one full day or even longer. When large cloud services become unavailable, there is a nearly instant and worldwide coverage of the outage. Such services as Amazon, Google, Citrix, etc. have experienced highly publicized outages during the past couple of years [Hoover 08b][InformationWeek 08a].

The users of a cloud service should exercise prudence, and take one or more of the following precautions. First, they should not entrust absolutely mission-critical applications and data to the cloud service providers; that is, they should use cloud services for non-missioncritical applications and data. This explains the current uses of cloud services for Web site hosting, software testing and online data backup. Second, they should keep backups of applications and data on on-premises servers and storage, or on a secondary cloud service. Third, they should secure as favorable a service-level agreement (SLA) as possible from the cloud service provider for a favorable partial redress in case of temporary outages [InformationWeek 08c]. We note that none of these precautions is entirely satisfactory. The first limits the use of the cloud service. The second and third erode the cost advantage of the cloud service. The third never fully compensates for the actual damage.

\section{Security}

The security of computer systems, and the data stored on them, can be compromised in so many ways, $100 \%$ security is simply impossible. Sophisticated hackers can break into just about any computer system. A cloud may become a "honey pot" that attracts hackers. Accidents may happen during physical transportation or electronic transfer of a large volume of data. Dishonest staff members may do bad things to the computer system or data.

We believe, however, that the clouds are not less secure than on-premises computing systems. There is no reason that the best security technologies and processes that can have been adopted for on-premises computing systems cannot be used by the cloud service providers. Further, the effects of security breaches on the cloud service providers are as great or even greater than those on medium to large organizations. As such, the cloud service 
providers should be highly motivated to do their best to secure their servers and data.

The security measures that Amazon Web Services employs may serve as a model for other cloud service providers. The user can run his customized machine image with a full root access. The user can have his own ingress firewall. The user can also have granular access control for every file. The user needs CERT, a public key, a long ID string, a security ID to access Amazon's resources [gnucitizen 08].

\section{Performance}

A major source of performance problem for cloud services is the communication time between the client computer and the Web server in the cloud. This problem becomes serious as the number of simultaneous users increases, and the amount of data transferred to and from the cloud increases. Even the physical distance between the client computer and the cloud makes a difference.

Sometimes organizations discover the need to substantially increase the communication bandwidth shortly after adopting cloud services [InformationWeek 08c]. Before adopting cloud services, organizations must assess the communication bandwidth requirements, and evaluate the performance behavior of the applications with respect to transfer of large amounts of data.

\section{Compliance}

In the US, enterprises are subject to some government regulations regarding the secure storage, privacy, and disclosure of data. The regulations include Sarbanes-Oxley Act for corporate accounting data, and HIPAA (Health Insurance Portability and Accountability Act) for people's healthcare insurance data. These regulations were written without consideration of cloud computing, that is, an enterprise storing data on a third-party computing facilities that are shared with other enterprises. It is not clear if cloud computing will violate such regulations.

\section{Private Clouds}

A private cloud is an on-premises cloud [Foley 08][InformationWeek 09][Weier 09]. A private cloud, except for its physical location, works just like a normal, or public, cloud. The virtual machines and storage are created by virtualizing physical computing resources; and the virtual computing resources are dynamically allocated and deallocated based on the needs of the users. Further, the users or departments in the enterprise are charged for the services they actually use. 
Since the term "cloud" was coined to refer to a remote third-party service provider, the term "private cloud" is an oxymoron. Besides, one of the primary motivations for cloud services has been touted as the freedom from having to administer on-premises computing resources. A possible justification for the use of the term private clouds is the fact that a private cloud is envisioned as a central cloud for an enterprise, and it is to be accessed by users in different departments, as though it is a remote computing resource. In any case, the concept of private clouds has gained grounds recently.

Private clouds can serve as a halfway step before the adoption of public cloud services. Enterprises can gain experience using cloud services, and prepare their IT infrastructure and staff properly. Further, enterprises can make use of hybrid cloud services based on their private clouds and some public clouds. For example, when the capacity of the private cloud is exceeded, the enterprise may tap into the public cloud.

There are adoption issues for hybrid cloud services. Today, if a workload is to be moved from a private cloud to a public cloud, both clouds require the same hypervisor, the same chipsets for the servers, and the same file system. Further, virtualization vendors have different virtual machine formats. To alleviate this problem, the Distributed Management Task Force has proposed an Open Virtual Machine Format.

\section{Integration}

Since organizations may need to adopt multiple service providers to various reasons, they need to integrate applications and data on multiple public clouds. Further, many organizations are likely to adopt hybrid clouds, they need to integrate applications and data between the private clouds and the public clouds [InformationWeek 08b][Schwartz 08]. Technologies such as enterprise information integration (or federated database systems), enterprise application integration, and enterprise service bus can be adapted to address the cloud integration issues.

\section{Cost}

Cost is generally not regarded as an adoption issue. People take the "only pay for what you use" part of the marketing definition of cloud computing as a given. In the 1980s and 1990s, people took for granted about the promise of cost savings in outsourcing software development from the US or Japan to countries such as India, China, etc. The cost savings, while still significant, turned out to be much less than had been presumed, because of the need to communicate between the two parties (e.g., travel, stationing staff), to re-do work that was not done properly, gradual increase in fees charged, etc. 
Similarly, the promised cost benefits of cloud computing are bound to be eroded. As observed above, the need to maintain on-premises backup or secondary cloud services in order to cushion the impact of occasional outages certainly adds to the cost. The need to increase communication bandwidth to maintain a desired performance level adds to the cost.

Further, the "remote administering of computing resources" part of the marketing definition of cloud computing does not mean that organizations that adopt cloud services can totally depend on the service providers for the administering of the applications, virtual machines and storage. The organizations still need to monitor the performance and availability of the virtual computing resources. There are various monitoring tools, both commercial and open source. Monitoring requires staff time, and possibly commercial tools. These add to the cost.

laaS service providers create virtual computing resources out of physical computing resources, and allocate the virtual computing resources to different users. This means that multiple users share common physical computing resources. Some organizations insist on having dedicated physical computing resources in the cloud in order to prevent other "tenants" from possibly crossing paths. The use of dedicated physical resources in the cloud can substantially erode the cost benefits of cloud computing.

\section{Conclusion}

In this paper, we examined various issues that (will) impede rapid adoption of cloud computing. The early euphoric expectation of huge cost benefits will surely be tempered. Even the definition of cloud computing will undergo refinement. It has already been changed to allow for private clouds, and payment schemes that include annual contracts. Further, such issues as outage and security are not likely to be fully resolved for all users.

However, we expect that cloud computing will become an important and viable step in the evolution of information technology. We expect that laas services will be dominated by a fairly small number of large vendors, such as Amazon, Google, IBM, HP, Sun, etc. However, there will surely be a very large number of SaaS service providers. There will also be a large number of private clouds. A new IT ecosystem for cloud computing will emerge that will include laaS service providers; SaaS, CaaS and PaaS service providers, some of which will provide their services on the virtual infrastructure they will buy from laas service providers; cloud management service providers, including cloud monitoring and cloud integration services; system integration service providers; consultancies and training service providers; etc.

\section{Acknowledgments}




\section{References}

[Babcock 09a] Charles Babcock. "Why 'Private Cloud' Computing Is Real - And Worth Considering," InformationWeek, April 11, 2009.

[Babcock 09b] Charles Babcock. "Hybrid Clouds: No Easy Concoction," InformationWeek, September 5, 2009.

[blogspot] http://cloudcomputing.blogspot.com/Cloud computing: adoption fears and strategic innovation opportunities",

[Foley 08] John Foley: "Private Clouds Take Shape", InformationWeek, August 9, 2008.

[gnucitizen 08] "The Cloud Is Not That Insecure," http://www.gnucitizen.org/blog/the-cloud-isnot-that-insecure'

[Gruman 08] Galen Gruman: "What Cloud Computing Really Means", InfoWorld, April 7, 2008. (Also in The New York Times, with Eric Knorr)

[Hoover 08a] J. Nicholas Hoover and Richard Martin: "Demystifying the Cloud", InformationWeek Research \& Reports, pp. 30-37, June 23, 2008.

[Hoover 08b] J. Nicholas Hoover: "Outages Force Cloud Computing Users To Rethink Tactics", InformationWeek, August 16, 2008.

[George 08] Randy George: “Cloud Storage’s Top Uses”, InformationWeek, October 20, 2008.

[InformationWeek 08a] "Amazon's S3 Cloud Service Turns Into A Puff of Smoke", InformationWeek NewsFilter, August, 2008.

[InformationWeek 08b] "Critical Link," InformationWeek Research \& Reports, October 20, 2008.

[InformationWeek 08c] "How to Plug Into the Cloud," InformationWeek Research \& Reports, December 8, 2008

[InformationWeek 09] "Private Clouds on the Horizon," InformationWeek Analytics, April 13, 2009.

[Kim 09] Won Kim. "Cloud Computing: Today and Tomorrow," Journal of Object Technology, January/February 2009. 
[Naone 07] Erica Naone: "Computer in the Cloud", MIT Technology Review, September 18, 2007.

[Schwartz 08] Mathew Schwartz. "Integrating Your Cloud And On-Premises Applications," http://www.bmighty.com/shared/article/printableArticle.Src.jhtml?articlelD=2111002 17

[Weier 09] Mary Weier. "Amazon Launches Private Cloud Services", InformationWeek, August 26, 2009.

[Wikipedia] "Cloud Computing", http://en.wikipedia.org/wiki/Cloud_computing 